\title{
Autophagy is essential for the endothelial differentiation of breast cancer stem-like cells
}

\author{
ZIANG YAO, ZEQING YANG, FENGJIA CHEN, YUE JIANG, CHENGZHU FU, \\ YONG WANG, RONGHAO LU and HAIGE WU
}

School of Life Science and Technology, Dalian University, Dalian, Liaoning 116622, P.R. China

Received May 31, 2019; Accepted October 4, 2019

DOI: $10.3892 /$ ijmm.2019.4399

\begin{abstract}
Blood vessels serve an important role in tumor growth and metastasis, and recent studies have shown that certain tumor cancer stem cells may differentiate into endothelial cells and contribute to angiogenesis. In the present study, vascular endothelial growth factor (VEGF) was used to induce endothelial differentiation of breast cancer stem-like cells (BCSLCs), and methods including flow cytometry, western blotting and immunofluorescence were used to study the relationship between autophagy and the endothelial differentiation of BCSLCs. The results showed that BCSLCs could differentiate into endothelial cells under the induction of VEGF in vitro. Subsequently, the role of autophagy in the endothelial differentiation of BCSLCs was examined. Autophagic activity was measured during endothelial differentiation of BCSLCs, and the association between autophagy and endothelial differentiation was investigated using autophagy activators, autophagy inhibitors and autophagy related 5 (Atg5)-knockdown BCSLCs. Autophagy was increased during endothelial differentiation of BCSLCs, and there was a positive association between autophagy and endothelial differentiation. The ability of cells to undergo endothelial differentiation was reduced in BCSLCs with Atg5 knockdown. Therefore, autophagy was essential for endothelial differentiation of BCSLCs, and the findings of the present study may highlight novel potential avenues for reducing angiogenesis and improving treatment of breast cancer.
\end{abstract}

\section{Introduction}

Folkman (1) proposed that tumor growth was vascularization-dependent, as there were numerous antitumor studies focused on anti-angiogenesis. Angiogenesis is the process by

Correspondence to: Professor Haige Wu, School of Life Science and Technology, Dalian University, 10 Xuefu Street, Economic and Technological Development Zone, Dalian, Liaoning 116622, P.R. China

E-mail: haige-hu@163.com

Key words: breast cancer stem-like cell, angiogenesis, vascular endothelial cell, autophagy, differentiation which new capillaries are formed from existing blood vessels to supply the tumor, and it is hypothesized to be essential for the continued growth and metastasis of solid tumors (2). Therefore, preventing tumor angiogenesis may prevent tumor growth. However, the results of anti-angiogenic therapies for treating patients with cancer remain unsatisfactory $(3,4)$. An increasing number of studies have shown that the process of angiogenesis is far more complicated than initially expected (5). Aside from angiogenesis, there are other cellular mechanisms contributing to the generation of vasculature in tumors, including vasculogenic mimicry, bone marrow-derived vasculogenesis and cancer stem-like cell-derived vasculogenesis (6). Cancer stem-like cells have been reported to differentiate into vascular endothelial cells and contribute to the formation of a vascular system in tumors $(7,8)$. Therefore, cancer stem-like cell-derived vasculogenesis should be considered in anti-angiogenic therapies for cancer treatment.

Autophagy is a physiological process that captures and degrades intracellular erroneous proteins and damaged organelles to maintain intracellular metabolism (9). Numerous studies have shown that autophagy serves an important role in cell differentiation. For example, terminal differentiation of immune cells is highly dependent on functional autophagy (10-12). Nuschke et al (13) demonstrated that undifferentiated bone marrow mesenchymal stem cells do not employ autophagy despite the accumulation of non-degraded autophagic vacuoles; however, when cells are stimulated by osteogenic differentiation, the rate of autophagy also increases. The essential role of autophagy in differentiation has been demonstrated in hepatic stem/progenitor cells (14), cardiac stem cells (15) and neuronal cells (16).

Bussolati et al (17) reported that breast tumor stem/progenitor cells could differentiate into endothelial cells. In the present study, breast cancer stem-like cells (BCSLCs), characterized as $\mathrm{CD} 44^{+} \mathrm{CD} 24^{-/ \text {low }}$, were isolated from MCF-7 breast cancer cells and induced to differentiate into endothelial cells using vascular endothelial growth factor (VEGF), and the association between autophagy and endothelial differentiation of BCSLCs was examined.

\section{Materials and methods}

Cells. MCF-7 cells were purchased from the China Center for Type Culture Collection and cultured in DMEM/F12 
medium (Gibco; Thermo Fisher Scientific, Inc.) supplemented with 10\% FBS (Gibco; Thermo Fisher Scientific, Inc.) and $1 \%$ penicillin-streptomycin (Sangon Biotech Co., Ltd.) at $37^{\circ} \mathrm{C}$ in $5 \% \mathrm{CO}_{2}$ atmosphere. Human umbilical vein endothelial cells (HUVECs; cat. no. ATCC ${ }^{\circledast}$ CRL-1730 ${ }^{\mathrm{TM}}$; American Type Culture Collection) supplemented with F-12K medium (cat. no. ATCC 30-2004 ${ }^{\mathrm{TM}}$; American Type Culture Collection) supplemented with $10 \%$ FBS (Gibco; Thermo Fisher Scientific, Inc.), $1 \%$ penicillin-streptomycin (Sangon Biotech Co., Ltd.), $0.1 \mathrm{mg} / \mathrm{ml}$ heparin (cat. no. H3393; Sigma-Aldrich; Merck KGaA) and $1 \%$ endothelial cell growth supplement (cat. no. 354006; BD Biosciences) at $37^{\circ} \mathrm{C}$ in $5 \%$ $\mathrm{CO}_{2}$. Pre-made lentiviral particles expressing a fusion target of green fluorescent protein (GFP)-red fluorescent protein (RFP)-LC3 (cat. no. JM-1314L204H-S) were purchased from Shanghai Genomeditech Co. Ltd. and transfected into MCF-7 cells according to the manufacturer's protocol. In brief, $1 \times 10^{4}$ MCF-7 cells/well were plated in a 6-well plate and cultured for $12 \mathrm{~h}$ to ensure that the cell density reached $30-50 \%$ when the virus was infected the following day. Health Gene transgene reagent (Health Gene Technologies) and $3 \times 10^{8} \mathrm{TU} / \mathrm{ml}$ lentivirus were used to transfect MCF-7 cells; $6 \mu \mathrm{g} / \mathrm{ml}$ polybrene was added to promote the transfection. After culture for $24 \mathrm{~h}$, the medium was renewed and cultured for another $8 \mathrm{~h}$, then $1.5 \mu \mathrm{g} / \mathrm{ml}$ puromycin (Sigma-Aldrich; Merck KgA) was added to isolate resistant cells. Then, monoclonal transfected cells were obtained and used for subsequent experiments.

Isolation of BCSLCs. BCSLCs, defined as $\mathrm{CD} 44^{+} \mathrm{CD} 24^{- \text {/low }}$ MCF-7 cells, were isolated using a Miltenyi Microbead kit (MiltenyiBiotec $\mathrm{GmbH}$ )accordingtothe manufacturer'sinstructions and cultured in stem cell medium, comprising serum-free medium supplemented with $20 \mathrm{ng} / \mathrm{ml}$ epidermal growth factor (EGF; Gibco; Thermo Fisher Scientific, Inc.), $20 \mathrm{ng} / \mathrm{ml}$ basic fibroblast growth factor (bFGF; Gibco; Thermo Fisher Scientific, Inc.) and $10 \mathrm{ng} / \mathrm{ml}$ B27 (Gibco; Thermo Fisher Scientific, Inc.). BCSLCs were grown to microsphere formation at $37^{\circ} \mathrm{C}$ with $5 \% \mathrm{CO}_{2}$ and observed under an inverted fluorescence microscope (magnification, x20; Leica DM IL LED Fluo; Leica Microsystems GmbH).

Flow cytometry. Sorted BCSLCs were cultured for $12 \mathrm{~h}$ in stem cell medium supplemented with $10 \mathrm{ng} / \mathrm{ml}$ VEGF, and VEGF-induced BCSLC and control BCSLCs were analyzed using flow cytometry. Cells were collected and adjusted to a cell density of $5 \times 10^{5}$ cells/tube. Cells were centrifuged at $300 \mathrm{x}$ g for $10 \mathrm{~min}$ at $4^{\circ} \mathrm{C}$, the supernatant was discarded, and cells were resuspended in $100 \mu \mathrm{l}$ buffer plus the corresponding antibody (1:20), and incubated at $4^{\circ} \mathrm{C}$ for $8 \mathrm{~min}$ in the dark. Subsequently, the cells were washed with $1 \mathrm{ml}$ buffer and centrifuged at $300 \mathrm{xg}$ for $10 \mathrm{~min}$ at $4^{\circ} \mathrm{C}$. The supernatant was discarded, cells were resuspended in $500 \mu \mathrm{l}$ buffer, stored in the dark on ice, and flow cytometry was performed within $30 \mathrm{~min}$. The following primary antibodies were used: Fluorescein isothiocyanate (FITC)-conjugated anti-human CD44 (cat. no. 130-113-903; Miltenyi Biotec GmbH), phycoerythrin (PE)-conjugated anti-human CD24 (cat. no. 130-098-861; Miltenyi Biotec $\mathrm{GmbH}$ ) PE-conjugated anti-human CD31 (cat. no. 130-110-807; Miltenyi Biotec GmbH) and FITC-conjugated anti-human CD105 (cat. no. 130-098-778;
Miltenyi Biotec $\mathrm{GmbH}$ ). Flow cytometry was performed using a FACSCalibur flow cytometer (BD Biosciences). The flow cytometry analysis software used was FlowJo ${ }^{\mathrm{TM}}$ v10.6.1 (BD Biosciences).

Matrigel tube formation assay. The growth and differentiation status of BCSLCs in vitro was evaluated by a Matrigel formation experiment. Matrigel (BD Biosciences) was thawed, and the blot was added to a 96 -well plate at $40 \mu 1 /$ well, with 3 replicate wells. The 96 -well plate was then placed in a $37^{\circ} \mathrm{C}$ incubator for $45 \mathrm{~min}$. After the gel had coagulated, MCF-7 cells and BCSLCs were seeded onto Matrigel at a density of $10^{4}$ cells/well. The cells were cultured for $12 \mathrm{~h}$ in complete or stem cell medium supplemented with or without $10 \mathrm{ng} / \mathrm{ml}$ VEGF. Tube formation was observed under an inverted microscope (magnification, x10; Olympus Corporation).

Griess method for detecting nitric oxide (NO) content. A total of $5 \times 10^{4}$ cells/well were plated in a 6-well plate, and cultured in stem cell medium supplemented with or without $10 \mathrm{ng} / \mathrm{ml}$ VEGF. After 3 days, the cell were $\sim 80 \%$ confluent and sub-cultured. After passaging, the cells were considered second generation of differentiation. The second-generation cell culture solution was used to detect the NO content. The content of $\mathrm{NO}$ in the supernatant was measured using the Griess assay protocol as previously described (18). The NO content in HUVEC culture medium was used as the control.

Confocal microscopy of LC3. mRFP-GFP-LC3 lentivirus was purchased from Shanghai Genomeditech Co. Ltd. MCF-7 cells were infected with lentivirus as aforementioned and transfected cells were selected for with $1.5 \mu \mathrm{g} / \mathrm{ml}$ puromycin. mRFP-GFP-LC3-transformed BCSLCs were isolated using a Miltenyi Microbead kit. The selected mRFP-GFP-LC3 double-labeled BCSLCs were seeded at a density of $1 \times 10^{4}$ cells/well in complete culture medium without VEGF, or stem cell culture solution with or without $10 \mathrm{ng} / \mathrm{ml}$ VEGF. The localization of RFP and GFP was observed using a confocal laser scanning microscope (Olympus Corporation) at x100 or x600 magnification.

Reverse transcription-quantitative (RT- $q) P C R$ analysis. The expression of CD105 was detected via RT-qPCR. Total RNA was extracted from cells using TRIzol extraction reagent (Takara Bio, Inc.). For reverse transcription, a PrimeScript ${ }^{\mathrm{TM}}$ Reverse Transcription kit (Takara Bio,Inc.) was used according to the manufacturer's instructions. qPCR was performed using SYBR Premix Ex Taq with Tli RNaseH Plus (Takara Bio, Inc.) according to the manufacturer's instructions. GAPDH was used as the internal reference. The PCR reaction conditions consisted of pre-denaturation at $95^{\circ} \mathrm{C}$ for $30 \mathrm{sec}$, followed by 40 cycles of denaturation at $95^{\circ} \mathrm{C}$ for $5 \mathrm{sec}$ and extension at $59^{\circ} \mathrm{C}$ for $30 \mathrm{sec}$. The sequence of the qPCR primers were as follows: CD105, forward 5'-AGAGGACAGGGGTGACAA GGT-3', reverse 5'-AAGTGTGGGCTGAGGTAGAGG-3'; and GAPDH, forward 5'-AGAAGGCTGGGGCTCATTTG-3', reverse 5'-AGGGGCCATCCACAGTCTTC-3'. Expression was measured using the $2^{-\Delta \Delta C q}$ method (19).

For the evaluation of endothelial differentiation, after sorting, cells were seeded at $2 \times 10^{5}$ cells/well in 6-well plates 
and divided into four groups: Control group; $10 \mathrm{ng} / \mathrm{ml}$ VEGF, $10 \mathrm{ng} / \mathrm{ml}$ VEGF + $100 \mathrm{nM}$ rapamycin (RAPA; Sigma-Aldrich; Merck KGaA) or $10 \mathrm{ng} / \mathrm{ml}$ VEGF $+400 \mathrm{nM}$ bafilomycin A1 (Baf A1; Abcam). Cells were treated for $24 \mathrm{~h}$, and cell morphology and CD105 expression was evaluated.

RNA interference. Short hairpin (sh)-autophagy related 5 (Atg5) was inserted into a lentiviral vector (pGMLV-SC7 RNAi; Shanghai Genomeditech Co. Ltd.). The sequences of the shRNA were: Forward 5'-GCAGATGGACAGTTG CACACAC-3', reverse, 5'-AGGTGTTTCCAACATTGGCTC A-3'. The transfection of sh-Atg5 lentivirus into MCF-7 was conducted according to the same protocol as the transfection of GFP-RFP-LC3 lentivirus into MCF-7 cells described above. sh-Atg5 BCSLCs were isolated using a Miltenyi Microbead kit, seeded in 6-well plates at a density of $2 \times 10^{5}$ cells/well, and cultured for $24 \mathrm{~h}$ in stem cell medium with or without $10 \mathrm{ng} / \mathrm{ml}$ VEGF.

Western blot analysis. Western blot analysis was used to detect the expression of autophagy-associated proteins. Cells were seeded at a density of $2 \times 10^{5}$ cells/well in a 6 -well plate and treated according to the experimental design. After the end of the experiment, total protein was extracted using a Whole Protein Extraction kit (cat. no. BC3710; Beijing Solarbio Science \& Technology Co., Ltd.) and the protein concentration was determined using a Bicinchoninic Acid Protein Assay kit (cat. no. PC0020; Beijing Solarbio Science \& Technology Co., Ltd.). Proteins (20 $\mu \mathrm{g} / \mathrm{lane})$ were separated via $12 \%$ SDS-PAGE and transferred to a PVDF membrane. Membranes were blocked with 5\% skimmed milk for $1 \mathrm{~h}$ at room temperature, and subsequently incubated with primary antibodies $(1: 1,000)$ at $4^{\circ} \mathrm{C}$ overnight and incubated for $1 \mathrm{~h}$ at room temperature the following day. After washing with TBS- $0.05 \%$ Tween-20 (TBST), the membrane was incubated with secondary antibody $(1: 1,000)$ for $2 \mathrm{~h}$ at room temperature. The membrane was washed thoroughly with TBST and signals were visualized using enhanced chemiluminescence reagent (Beijing Solarbio Science \& Technology Co., Ltd.). Grayscale analysis was performed using Gel-Pro Analyzer V.4.0 (Meyer Instruments, Inc.). The primary antibodies used were $\beta$-actin (cat. no. 4970), LC3 (cat. no. 4108), p62 (cat. no. 5114), Beclin1 (cat. no. 3495), Atg3 (cat. no. 3415), Atg5 (cat. no. 12994), Atg7 (cat. no. 8558), Atg16L1 (cat. no. 8089), Unc-51-like autophagy activating kinase (ULK; cat. no. 8054), phosphorylated (p)-ULK (cat. no. 5869), AMP kinase (AMPK; cat. no. 5832) and p-AMPK (cat. no. 2535); the secondary antibody used was a horseradish peroxidase-conjugated goat anti-rabbit immunoglobulin G antibody (cat. no. 7074). All antibodies were purchased from Cell Signaling Technology, Inc.

To monitor autophagy flux during endothelial differentiation, after sorting, cells were seeded at $2 \times 10^{5}$ cells/well in 6-well plates and divided into three groups: $0 \mathrm{~h}$ VEGF; $24 \mathrm{~h}$ VEGF (10 ng/ml); and $24 \mathrm{~h}$ VEGF + $1 \mathrm{~h}$ chloroquine (CQ; $50 \mu \mathrm{M}$; Sigma-Aldrich; Merck KGaA).

Statistical analysis. Results are presented as the mean \pm standard deviation of at least three independent experiments. Data were analyzed by t-test, or by one-way ANOVA followed by Tukey's test post hoc analysis. Statistical analysis was performed using Graph-Pad Prism 6.01 software (GraphPad Software, Inc.). P $<0.05$ was considered to indicate a statistically significant difference.

\section{Results}

BCSLC sorting and microsphere culture. Cancer stem cells (CSCs) are a type of tumor cell that are capable of self-renewal and differentiation (20). A previous study showed that CSCs can survive in serum-free medium containing bFGF, EGF, B27 and other growth factors, and gradually form microspheres during culture (21). Cells characterized as $\mathrm{CD} 44^{+} \mathrm{CD} 24^{- \text {-low }}$ are considered to be breast cancer stem cells. First, CD24-llow cells were isolated by sorting using immunomagnetic beads, and the expression of CD24 was detected via flow cytometry. As presented in Fig. 1A, the proportion of cells characterized as CD24+ in MCF-7 was 53.69 and $46.28 \%$ were cells characterized as CD24-low; the two cell populations had notable partitions (Fig. 1Ab). The proportion of CD24-/low cells obtained using immunomagnetic beads was $99.61 \%$, indicating that the purity of the obtained CD24-llow type cell was high and could be used for the following isolations (Fig. 1Ad). CD $44^{+}$cells were further sorted from the isolated CD24-/low cells using immunomagnetic beads, and the results of flow cytometry showed that the proportion of $\mathrm{CD} 44^{+}$cells was $99.57 \%$ (Fig. 1B). The obtained CD44 ${ }^{+} \mathrm{CD} 24^{- \text {llow }}$ cells were considered BCSLCs, and the obtained BCSLCs were identified using flow cytometry; the results showed that the proportion of $\mathrm{CD} 44^{+} \mathrm{CD} 24^{- \text {llow }}$ cells in BCSLCs was $41.62 \%$ (Fig. 1Cc), considerably higher than the proportion of cells obtained from the MCF-7 cells (1.33\%; Fig. 1Cb). Therefore, the $\mathrm{CD} 44^{+} \mathrm{CD} 24^{- \text {/low }}$ cells were enriched using immunomagnetic beads. The obtained BCSLCs were sub-cultured, and the proportion of $\mathrm{CD} 44^{+} \mathrm{CD} 24^{-/ \mathrm{low}}$ cells decreased to $10.93 \%$ when cultured up to passage 8 (Fig. 1Ce).

Microspheres gradually formed when BCSLCs were cultured in serum-free medium containing bFGF and EGF. The obtained BCSLCs with CD $44^{+} \mathrm{CD} 24^{- \text {/low }}$ specificity were cultured in stem cell culture medium, and the results showed that microspheres gradually formed when the cells were cultured for $64 \mathrm{~h}$ or more (Fig. 1D), suggesting that the obtained BCSLCs exhibited characteristics associated with cancer stem cells.

Endothelial differentiation of BCSLCs in vitro. VEGF was used as an inducer of endothelial differentiation. MCF-7 and BCSLCs were seeded on Matrigel and cultured for $12 \mathrm{~h}$ in stem cell medium with or without VEGF to mimic the growth and differentiation of cells in vivo. As presented in Fig. 2A, after incubation with VEGF-containing stem cell culture medium, BCSLCs formed a tubular structure, whereas MCF-7 cells did not.

$\mathrm{NO}$, which is an endothelium-dependent relaxation factor, is secreted by vascular endothelial cells expressing active NO synthase (22). The release of NO can be used as an indicator of endothelial cell viability (23). The results showed that BCSLCs treated with VEGF released significantly higher quantities of NO compared with cells not treated with VEGF (Fig. 2B). The quantity of NO released by induced BCSLCs was higher compared with the levels released by HUVECs. Thus, BCSLCs exhibited a property typically associated with endothelial cells when induced by VEGF. 

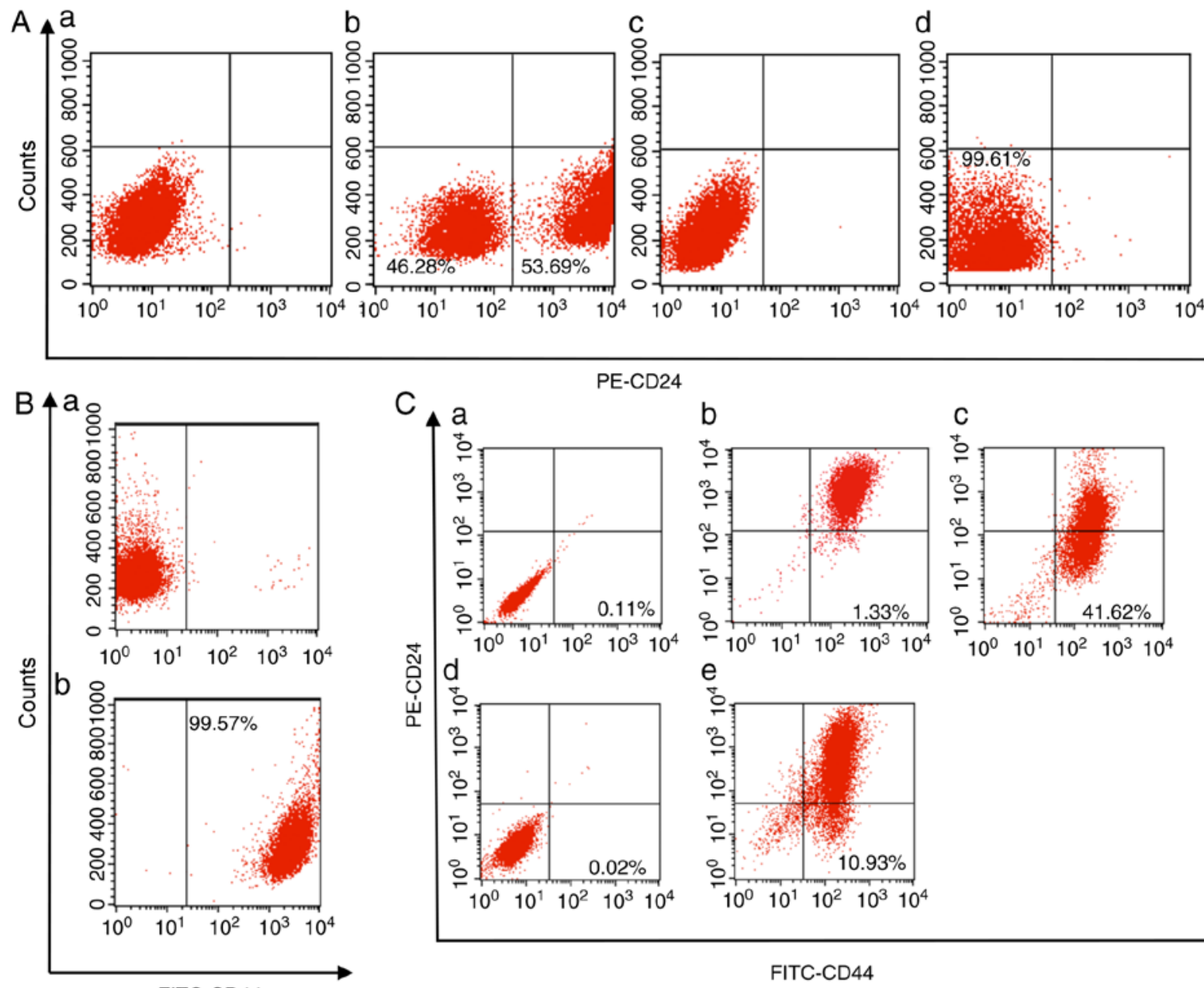

FITC-CD44

FITC-CD44

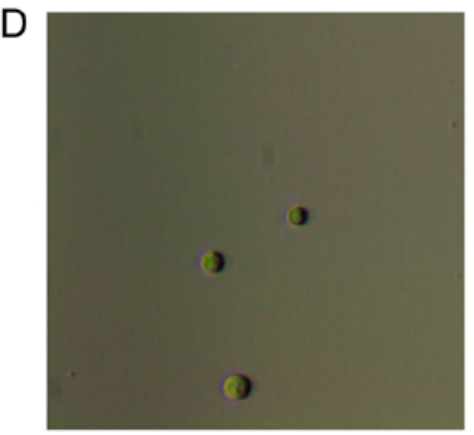

$\mathrm{Oh}$

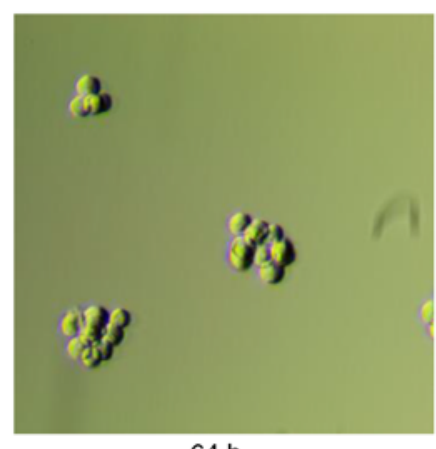

$64 \mathrm{~h}$

Figure 1. Flow cytometry and microsphere culture of BCSLCs. (A) CD24-/low cells were isolated from MCF-7 using immunomagnetic beads, and the expression of CD24 in MCF-7 and isolated CD24-llow cells were assessed using flow cytometry. (a) Isotype control for (b); (b) MCF-7 cells; (c) isotype control for (d); (d) isolated CD24-/low cells. (B) CD44 ${ }^{+}$cells were further isolated from CD24-/low cells and the expression of CD44 was assessed using flow cytometry. (a) Isotype control for (b); (b) CD44+ cells. (C) Expression of CD24 and CD44 in MCF-7, BCSLCs and BCSLCs after eight passages. (a) isotype control for (b); (b) MCF-7 cells; (c) CSLCs; (d) isotype control for (e); (e) BCSLCs after eight passages. (D) The isolated BCCSLCs were cultured in microspheres for 0 and $64 \mathrm{~h}$ in stem cell culture medium. BCSLC, breast cancer stem-like cell; PE, phycoerythrin.

CD31 and CD105 are used as markers of endothelial cells (24). The BCSLCs were cultured with or without VEGF for $12 \mathrm{~h}$, and the expression of CD105 and CD31 was assessed using flow cytometry. The results showed that in induced cells, the proportion of cells expressing CD105 and CD31 was 78.45 and $50.44 \%$, respectively, significantly higher compared with cells not treated with VEGF (Fig. 2C). These results suggested that after being induced by VEGF, BCSLCs expressed endothelial cell markers.
Alterations in autophagy during endothelial differentiation of BCSLCs. Autophagy is a dynamic physiological metabolic process in the cell. Detection of autophagy flow can more accurately reflect the changes of autophagy metabolism (25). Chloroquine (CQ) is used as an autophagy inhibitor, as it can alter the $\mathrm{pH}$ of the lysosome to abrogate lysosomal function, and inhibit binding of the autophagosome to the lysosome, thus blocking autophagy and resulting in an accumulation of autophagosomes (26). To monitor autophagy flux during 


\section{A}

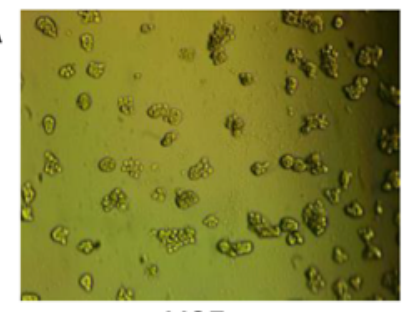

MCF-7

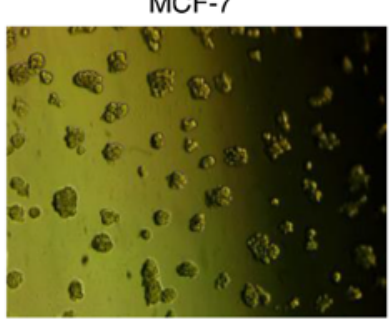

BCSLCs

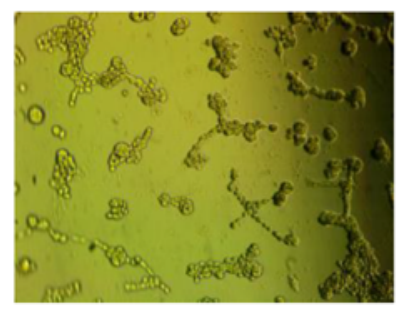

Induced MCF-7

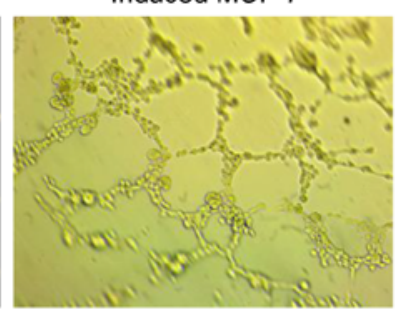

Induced BCSLCs
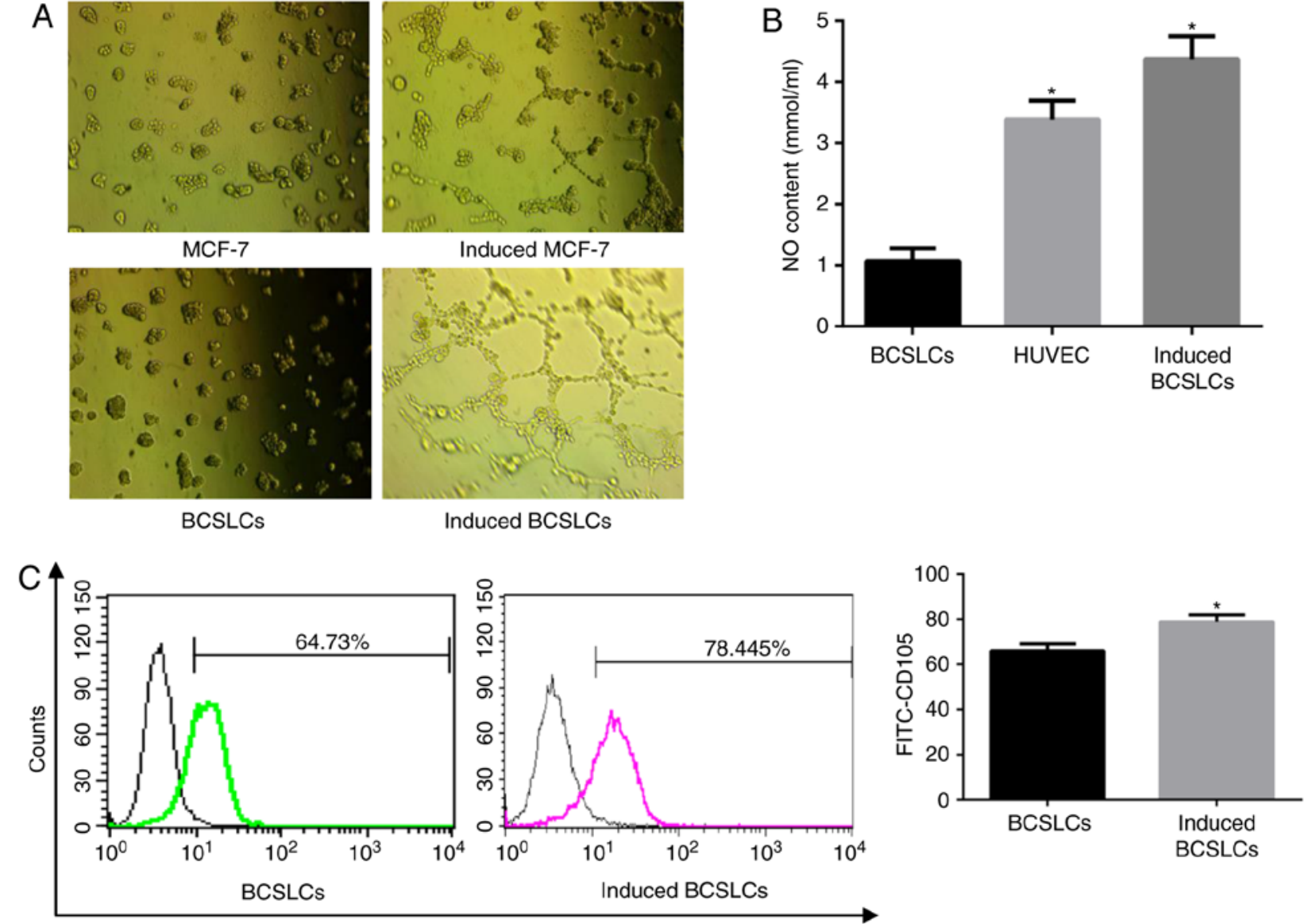

FITC-CD105
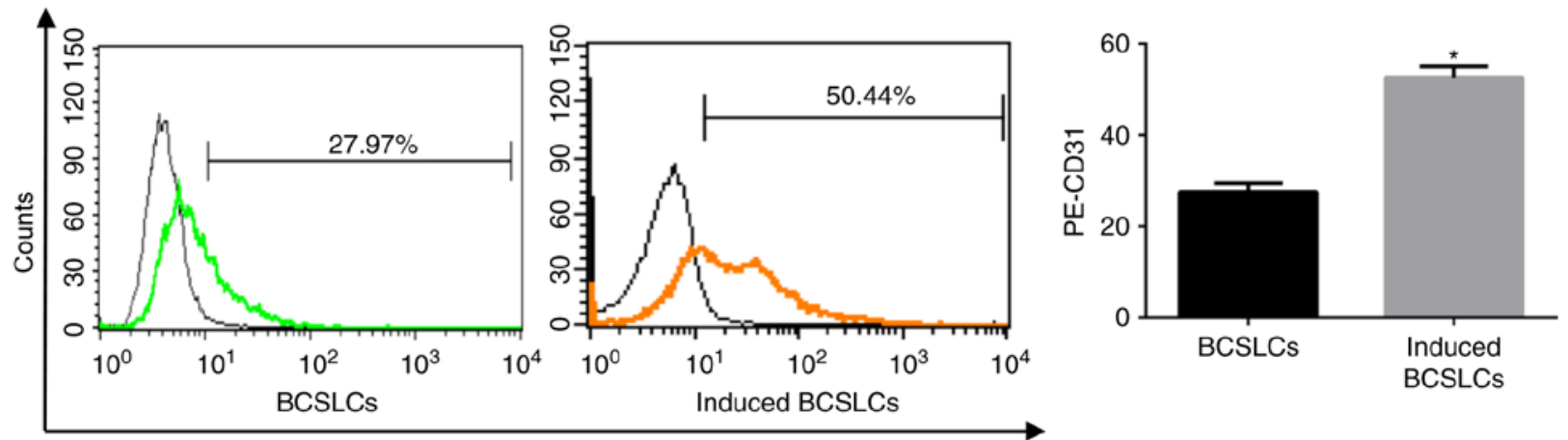

PE-CD31

Figure 2. BCSLCs can be induced to differentiate into endothelial cells by VEGF. (A) MCF-7 cells and BCSLCs were seeded on Matrigel and cultured for $12 \mathrm{~h}$ in stem cell medium with or without VEGF. (B) BCSLCs were cultured in stem cell medium with or without VEGF for $12 \mathrm{~h}$ as the experimental group and HUVECs were cultured in complete medium for $12 \mathrm{~h}$ as the positive control group, and the amount of NO released was detected. Data were analyzed by one-way ANOVA followed by Tukey's test post hoc analysis; "P<0.05 vs. BCSLCs. (C) BCSLCs were cultured for $12 \mathrm{~h}$ in stem cell medium with or without VEGF, and the expression levels of CD105 and CD31 were assessed using flow cytometry. Data were analyzed by t-test; ${ }^{*} \mathrm{P}<0.05$ vs. BCSLCs. BCSLC, breast cancer stem-like cell; HUVEC, human umbilical vein endothelial cell; NO, nitric oxide; VEGF, vascular endothelial growth factor.

endothelial differentiation of BCSLCs, CQ was used to inhibit autophagy, and the expression of Beclin1, p62 and LC3-II was detected via western blotting. The results showed that the expression levels of Beclin1,p62 and LC3-II were significantly increased when the BCSLCs were induced by VEGF for $24 \mathrm{~h}$ (Fig. 3A), suggesting that autophagy was increased. When the BCSLSs were treated with CQ for $1 \mathrm{~h}$ following treatment with VEGF, the expression of LC3-II and p62 was increased significantly, and the expression of Beclin1 was decreased significantly compared with cells not treated with CQ (Fig. 3A). Treatment with CQ would result in the accumulation of autophagosomes, which would reduce expression of upstream Beclin1 expression via negative feedback regulation (27). The accumulation of LC3-II reflects the increase in autophagy flux when induced by VEGF.

MCF-7 cells stably expressing mRFP-GFP-LC3 were created to further examine the association between autophagy and endothelial differentiation of BCSLCs. Fig. 3B shows the sequencing results. The sequence of the inserted fragment in the recombinant clone was completely identical to the sequence of the target fragment, and thus the construction of the overexpression vector was successful. Fluorescent 


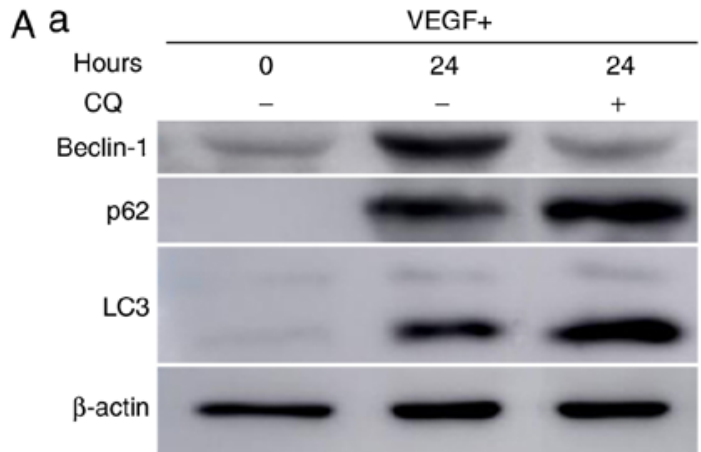

$\mathrm{B}$ a

\begin{tabular}{c|c} 
Plasmid & CMV-TURBORFP-EGFP-M_LC3-PGK-PUAO \\
\hline CCATGCCGTCCGAGAAGACCTTCAAGCAGCGCCGGAGCTTTGAACAAAGAGTGGAA
\end{tabular} CCATGCCGTCCGAGAAGACCTTCAAGCAGCGCCGGAGCITTGAACAAAGAGTGGAA
GATGTCCGGCTCATCCGGGAGCAGCACCCCACCAAGATCCCAGTGATATAGAGCG ATACAAGGGGGAGAAGCAGCTGCCCGTCCTGGACAAGACCAAGTTCCTGGTGCCTC GCTAACCAAGCCTTCTTCCTCCTGGTGAATGGGCACAGCATGGTGAGTGTGTCCAC CCCATCTOCGAAGTGTACGAGAGTGAGAGAGATGAAGACGGCTTCCTGTACATGGT TTATGCCTCGCAGGAGACATTCGGGACAGCAATGGCTGTGTAA

b

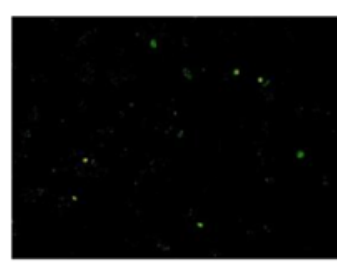

TurboRFP-EGFP-LC3

D

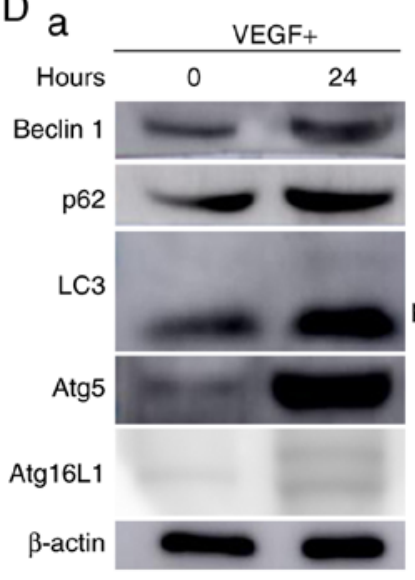

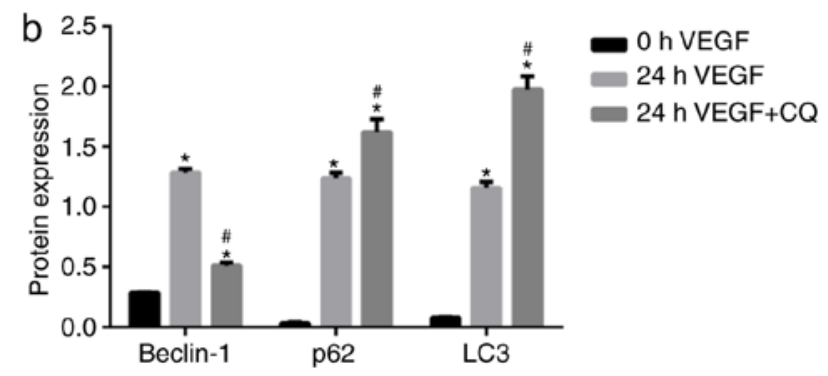

C

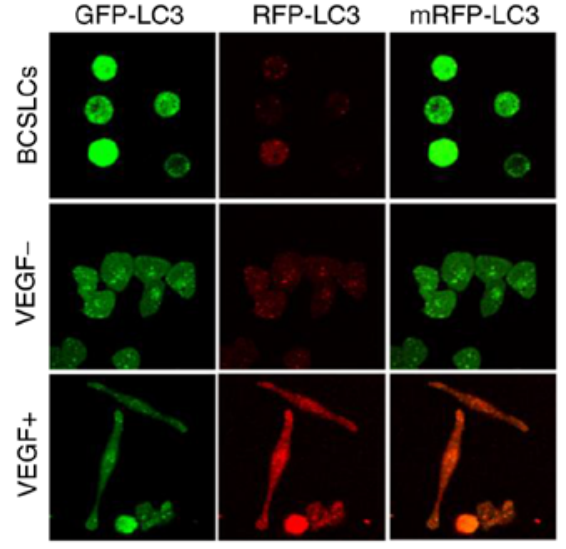

b

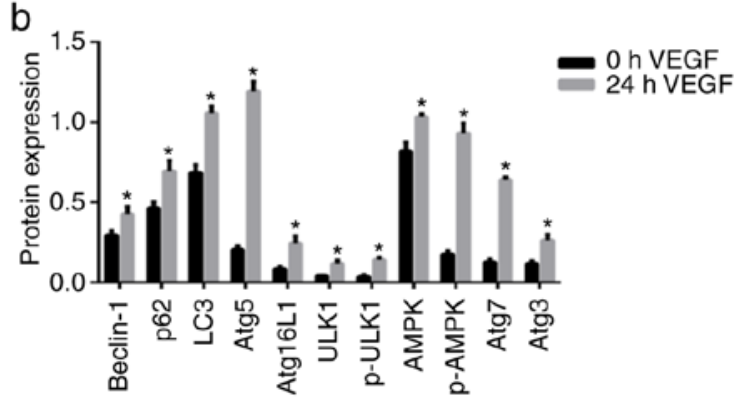

C

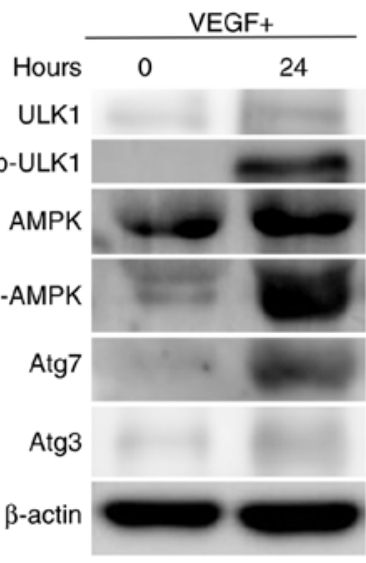

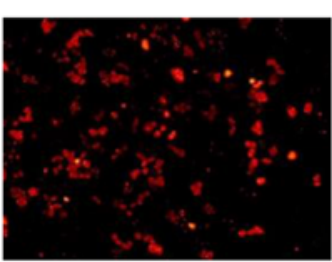

TurboRFP-EGFP-LC3

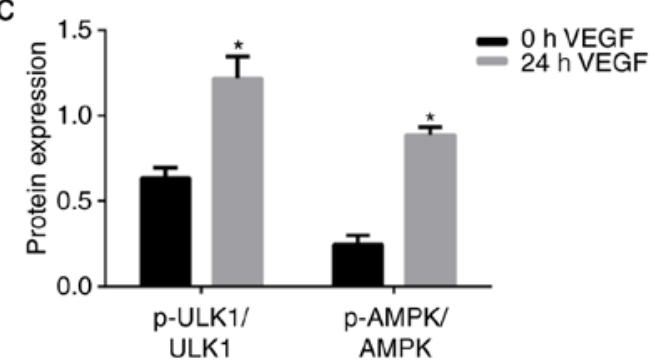

Figure 3. Alterations in autophagy activity during endothelial differentiation of BCSLCs. (A) BCSLCs were cultured in stem cell culture medium containing VEGF for 0 and $24 \mathrm{~h}$, with or without CQ. Western blotting was used to detect the expression of Beclin1, p62 and LC3-II. (a) Example western blot figure, (b) grayscale analysis. Data were analyzed by one-way ANOVA followed by Tukey's test post hoc analysis; ${ }^{*} \mathrm{P}<0.05 \mathrm{vs}$. $0 \mathrm{~h}$ VEGF group; ${ }^{\text {} P} \mathrm{P}<0.05 \mathrm{vs}$. $24 \mathrm{~h}$ VEGF group. (B) MCF7 cells transfected with RFP-GFP-LC3. (Ba) Sequencing results of the RFP-GFP-LC3 plasmid. (B-b) Fluorescent expression of GFP and RFP in MCF7 cells following transfection. (C) BCSLCs were cultured in complete medium or stem cell medium with or without VEGF for 24 h, and the RFP and GFP intensity were observed under a confocal laser scanning microscope. (D) BCSLCs were cultured in stem cell culture medium containing VEGF for 0 and $24 \mathrm{~h}$, and the expression of autophagy signaling pathway-associated proteins were detected using western blotting. (a) Example western blot figure, (b and c) grayscale analysis. Data were analyzed by t-test; ${ }^{*} \mathrm{P}<0.05$ vs. $0 \mathrm{~h}$ VEGF group. AMPK, AMP kinase; Atg, autophagy related; BCSLC, breast cancer stem-like cell; CQ, chloroquine; GFP, green fluorescent protein; p, phosphorylated; RFP, red fluorescent protein; ULK, Unc-51-like autophagy activating kinase; VEGF, vascular endothelial growth factor.

signals were detected after lentiviral infection, and GFP and RFP were visible, demonstrating successful transfection of mRFP-GFP-LC3 in MCF-7 cells (Fig. 3B). BCSLCs were isolated from the RFP-GFP-LC3-MCF-7 cells, and cultured in complete medium, or stem cell medium with or without VEGF for $24 \mathrm{~h}$, and the cells were observed under a confocal laser scanning microscope (Fig. 3C). After $24 \mathrm{~h}$ of cell culture, the green fluorescence intensity of the VEGF-containing group was lower compared with stem cell culture cells. There was an increase in the number of red and yellow spots in the cells cultured in the VEGF-containing stem cell culture medium compared with cells cultured in the complete medium. LC-3 
has two forms, LC3-I and LC3-II. LC3-I is diffusely distributed in cells, and LC3-II accumulates on the inner and outer membranes of autophagic vesicles, including autophagy precursors, and autophagosomes, making these autophagic structures appear green in the transfected cells. When autophagosomes fuse with lysosomes, the green fluorescence should be quenched due to the low $\mathrm{pH}$, but the red fluorescence should remain stable (28). Therefore, RFP was used to track LC3, and the weakening of green fluorescence was used to indicate the fusion of autophagosomes and lysosomes. Autophagy flux of BCSLCs in stem cell culture medium containing VEGF was markedly higher compared with cells grown in stem cell culture medium or complete medium. BCSLCs were induced into a spindle shape akin to the typical shape of vascular endothelial cells when treated with VEGF. These data suggested that the initiation of transdifferentiation of BCSLCs was accompanied by an increase in autophagic flux.

Proteins associated with autophagy signaling pathways were detected by western blotting to further determine the changes in autophagy levels during the endothelial differentiation of BCSLCs. The results showed that treatment with VEGF significantly increased the expression levels of ULK1, p-ULK1, AMPK and p-AMPK, all of which are upstream of the autophagy signaling pathway; the expression levels of key proteins involved in the steps between autophagy formation to autophagic lysosome degradation, such as Beclin1, Atg5, Atg16L1, Atg7, Atg3 and p62, were also increased compared with BCSLCs (Fig. 3D). There results suggested that autophagy levels were increased overall during endothelial differentiation of BCSLCs induced by VEGF. The analysis of ULK1, p-ULK1, AMPK, and p-AMPK showed that the ratios of p-AMPK/AMPK and p-ULK1/ULK1 were also increased significantly (Fig. 3Dc), indicating that the AMPK protein was activated, which in turn affects downstream ULK1 expression and phosphorylation, thus initiating autophagy (25).

Regulation of autophagy affects endothelial differentiation of BCSLCs. In order to investigate the association between autophagy and endothelial differentiation of BCSLCs, the autophagy activator RAPA and inhibitor Baf A1 were used to treat BCSLCs separately. As shown in Fig. 4A, cells initially showed an elongated spindle-like shape, similar to endothelial cells when treated with VEGF. When the cells were treated with VEGF and RAPA, the cellular morphology was also elongated and spindle-like, but when the cells were treated with VEGF and $\mathrm{Baf} \mathrm{A} 1$, the cellular morphology was cobblestone-like similar to that of BCSLCs without VEGF. The results suggest a positive association between autophagy and endothelial differentiation of BCSLCs. Expression of CD105 was assessed using RT-qPCR, and the results showed that treatment with VEGF or VEGF + RAPA increased expression of CD105 significantly compared with BCSLCs not treated with VEGF, and there was no significant difference in CD105 expression levels between these two groups. However, the expression of CD105 was decreased significantly when BCSLCs were treated with VEGF + Baf A1 compared with the VEGF group (Fig. 4B). These results were in agreement with the cellular morphology experiments, and a positive association between autophagy and the endothelial differentiation of BCSLCs was further suggested.
As Atg5 is one of the core proteins involved in autophagosome formation, an Atg5-knockdown MCF-7 cell line was established to further investigate if autophagy was critical for endothelial differentiation of BCSLCs. sh-Atg5-BCSLCs were isolated from sh-Atg5-MCF-7 and were induced with VEGF for $24 \mathrm{~h}$, and the expression of Atg5 and LC3-II was assessed via western blotting. As shown in Fig. 5A, after being induced with VEGF, the expression levels of Atg5 and LC3-II were similar in the control and vector groups, but significantly downregulated in the sh-Atg5 group. This indicated that autophagy was inhibited in sh-Atg5-BCSLCs. When the sh-Atg5-BCSLCs and vector-BCSLCs (negative control) were cultured with $1 \mathrm{ng} / \mathrm{ml}$ VEGF for $24 \mathrm{~h}$, the vector-BCSLCs exhibited an elongated spindle-like morphology similar to endothelial cells; however, the majority of the sh-Atg5-BCSLCs retained their cobblestone-like morphology (Fig. 5B). CD105 mRNA expression levels were measured and the results showed that the expression of CD105 was significantly decreased in sh-Atg5-BCSLCs following treatment with VEGF compared with the vector-BCSLCs (Fig. 5C). All the above results suggested that autophagy is required for effective endothelial differentiation of BCSLCs.

\section{Discussion}

Since Folkman (1) suggested that blood vessels are necessary for the development and growth of solid tumors in 1971, there have been a large amount of data supporting this hypothesis (29). The notion that CSCs are cancer cells that cause tumor growth and metastasis was first proposed in leukemia and later in solid tumors, including breast cancer (30). Studies have shown that CSCs can differentiate into endothelial progenitor cells $(31)$, endothelial cells $(32,33)$ or vascular smooth muscle-like cells (34), highlighting an alternate means of tumor blood vessel formation, and provides a new target for anti-angiogenic therapy in tumor.

The presence of CSCs has been demonstrated in solid tumors such as the colon, liver, lung, and pancreas, and breast cancer stem cells (BCSCs) were the first CSCs to be reported in a solid tumor (35). Recent findings have identified different types of BCSCs with various markers (36). Among these, $\mathrm{CD} 44^{+} \mathrm{CD} 24^{-/ \text {low }}$ was most frequently used to characterize BCSCs since it was discovered by Al-Hajj et al (30) in 2003. In the present study, CD $44^{+} \mathrm{CD} 24^{-/ \text {low }}$ BCSLCs were isolated from MCF-7 using the magnetic activated cell sorting method, and the isolated BCSLCs exhibited stemness by forming microspheres in the stem cell culture medium. VEGF was used as an endothelial differentiation inducer, and the BCSLCs presented with a long spindle-like morphology, similar to endothelial cells, and formed tube-like structures. Additionally, the induced BCSLCs released increased quantities of NO, and these results showed that the induced BCSLCs behaved in a similar manner to endothelial cells. Flow cytometry showed that the induced BCSLCs expressed increased amounts of CD31 and CD105, both of which are endothelial cell-specific markers. All the above results indicated that BCSLCs can be induced to differentiate into endothelial cells by VEGF.

Previous studies have found that autophagy is associated with stemness maintenance and the differentiation of 
A

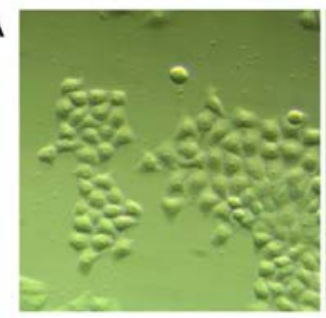

VEGF-

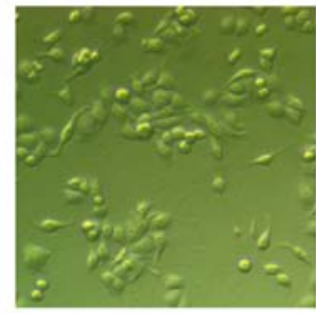

VEGF+RAPA

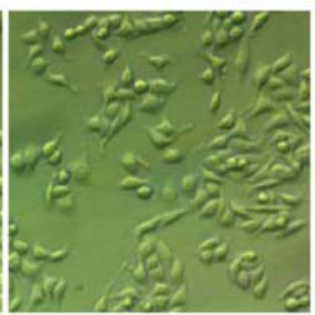

VEGF+

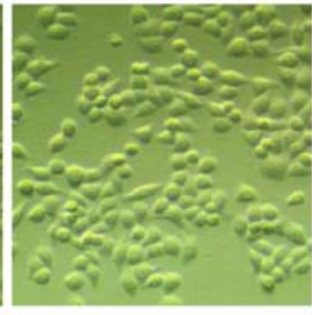

VEGF+Baf A1

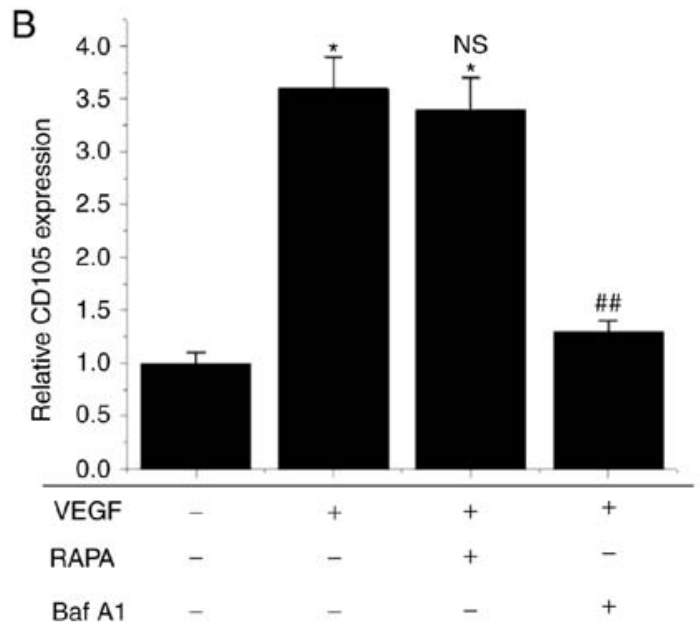

Figure 4. Regulation of autophagy affects the endothelial differentiation of BCSLCs. (A) BCSLCs were cultured in stem cell medium alone, with $10 \mathrm{ng} / \mathrm{ml}$ VEGF, with $10 \mathrm{ng} / \mathrm{ml}$ VEGF $+100 \mathrm{nM}$ RAPA, or with $10 \mathrm{ng} / \mathrm{ml} \mathrm{VEGF}+400 \mathrm{nM}$ Baf A1 for $24 \mathrm{~h}$. The morphology of the cells was observed under an inverted microscope. (B) mRNA expression levels of CD105 in BSCLCs grown in various culture mediums. Data were analyzed by t-test; ${ }^{P}<0.05$ vs. control group; ${ }^{\# \#} \mathrm{P}<0.01$ vs. VEGF group; ns, P>0.05 vs. VEGF group. Baf A1, bafilomycin A1; BSCLC, breast cancer stem-like cell; RAPA, rapamycin; VEGF, vascular endothelial growth factor.

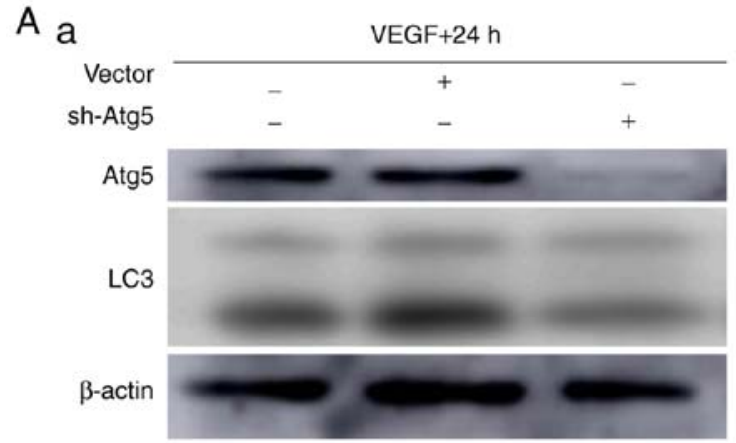

B

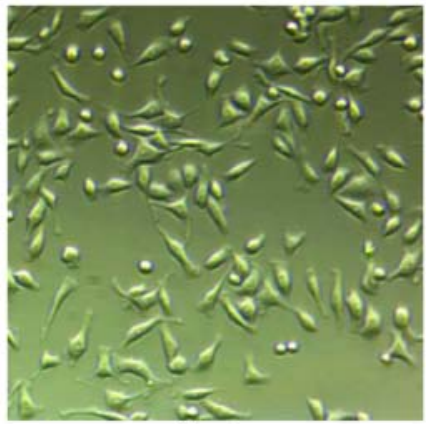

Vector

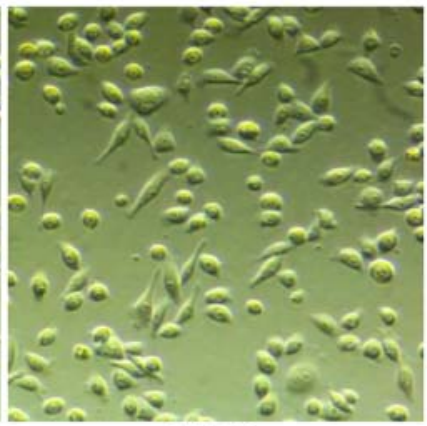

sh-Atg5
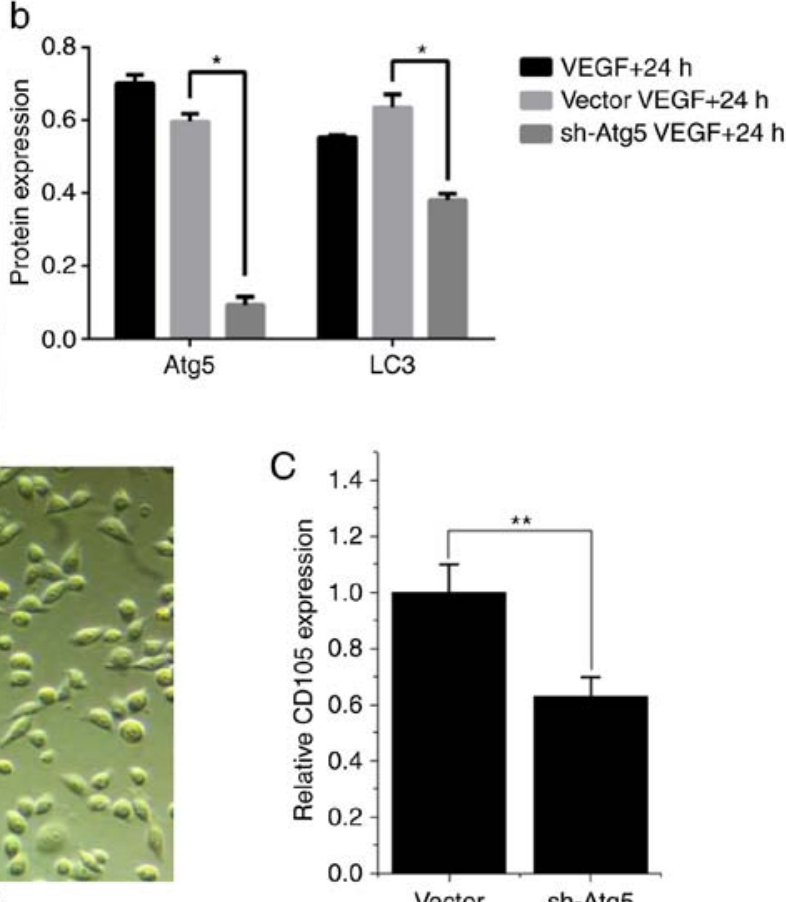

Vector sh-Atg5

Figure 5. Atg5 is required for the endothelial differentiation of BCSLCs. (A) Vector-BCSLCs and sh-Atg5-BCSLCs were cultured in stem cell medium containing VEGF for $24 \mathrm{~h}$, protein was extracted, and the expression of Atg5 and LC3 was detected via western blotting. Data were analyzed by one-way ANOVA followed by Tukey's test post hoc analysis; ${ }^{*} \mathrm{P}<0.05$. (a) Example western blot figure, (b) grayscale analysis. (B) Vector-BCSLCs and sh-Atg5 BCSLCs were cultured with VEGF for $24 \mathrm{~h}$, and the morphology of the cells was observed under an inverted microscope. (C) Expression of CD105 was detected via reverse transcription-quantitative PCR analysis. Data were analyzed by t-test; ${ }^{* *} \mathrm{P}<0.01$ vs. Vector. Atg, autophagy related; BSCLC, breast cancer stem-like cell; sh, short hairpin (RNA); VEGF, vascular endothelial growth factor.

stem cells. For example, in hepatocellular carcinoma, it was found that autophagy promoted cancer cell survival and resistance to chemotherapeutic drugs, as well as stem cell-like properties (37). Autophagy was reported to decrease reactive oxygen species generation and maintain stemness in mesenchymal stem cells (38). Notably,
Zhang et al (39) found that increased autophagy in cardiac stem cells promoted myocardial differentiation. Since then, there have been numerous studies demonstrating the requirement of autophagy for differentiation of stem/progenitor cells $(20,40)$. However, whether autophagy participated in the endothelial differentiation of BCSLCs 
was unknown. In the present study, LC3-II, Beclin1 and p62 were used as autophagy flux indicators, and it was shown that autophagy was increased significantly when BCSLCs were induced by VEGF for $24 \mathrm{~h}$, suggesting that autophagy participated in endothelial differentiation of BCSLCs. The RFP-GFP-LC3-BCSLCs were induced by VEGF, and an increase in red and yellow puncta, and weakened green fluorescence intensity were observed, indicative of enhanced autophagy flux during endothelial differentiation of BCSLCs. In the autophagy signaling pathway, the initiation of autophagy flux starts from the phosphorylation of ULK1, and the phosphorylation of AMPK can activate the phosphorylation of ULK1 $(25,41,42)$. Western blot analysis showed that after treatment with VEGF, the ratios of p-AMPK/AMPK and p-ULK1/ULK1 increased significantly, indicating that the AMPK protein was activated, which in turn affects downstream ULK1 expression and phosphorylation, initiating autophagy. Additionally, the expression levels of key proteins involved in autophagosome formation to autophagic lysosome degradation were increased notably.

Autophagy flux was upregulated during the endothelial differentiation of BCSLCs; however, whether autophagy was essential for the endothelial differentiation of BCSLCs remained to be demonstrated. RAPA and Baf A1 were used to activate or inhibit autophagy, respectively. VEGF could not induce BCSLCs to differentiate into endothelial cells if autophagy was inhibited by Baf A1. This result was further validated using BCSLCs with knockdown of Atg5. Atg5 is one of the key autophagy proteins, and Atg5-knockdown is commonly used to investigate the role of autophagy (43-46). In the present study, Atg5-knockdown BCSLCs could not be induced to differentiate into endothelial cells, indicating the important role of autophagy in endothelial differentiation of BCSLCs.

In conclusion, BCSLCs could be induced to differentiate into endothelial cells by VEGF in vitro, and autophagy participates in the endothelial differentiation of BCSLCs. There was a positive association between autophagy and the endothelial differentiation of BCSLCs, and autophagy was required for endothelial differentiation in BCSLCs. Therefore, autophagy may be a potential target for the treatment of breast cancer, and the present study provides a theoretical basis for the treatment of breast cancer with anti-angiogenic therapy by targeting autophagy.

\section{Acknowledgements}

Not applicable.

\section{Funding}

The present study was supported by the National Natural Science Foundation of China (grant. nos. 81373429 and 81671243).

\section{Availability of data and materials}

All data generated or analyzed during the present study are included in this published article.

\section{Authors' contributions}

ZYao and HW contributed to the conception and design of the study, and revised the manuscript. FC, ZYan and YJ designed and performed the experiments, and drafted the original manuscript. CF, YW and RL analyzed the data. All authors read and approved the final manuscript.

\section{Ethics approval and consent to participate}

Not applicable.

\section{Patient consent for publication}

Not applicable.

\section{Competing interests}

The authors declare that they have no competing interests.

\section{References}

1. Folkman J: Tumor angiogenesis: Therapeutic implications. N Engl J Med 285: 1182-1186, 1971.

2. Folkman J: What is the evidence that tumors are angiogenesis dependent? J Natl Cancer Inst 82: 4-6, 1990.

3. Bergers G and Hanahan D: Modes of resistance to anti-angiogenic therapy. Nat Rev Cancer 8: 592-603, 2008.

4. Sennino B and McDonald DM: Controlling escape from angiogenesis inhibitors. Nat Rev Cancer 12: 699-709, 2012.

5. Carmeliet P and Jain RK: Molecular mechanisms and clinical applications of angiogenesis. Nature 473: 298-307, 2011.

6. Jain RK and Carmeliet P: SnapShot: Tumor Angiogenesis. Cell 149: 1408-1408.e1, 2012.

7. Ricci-Vitiani L, Pallini R, Biffoni M, Todaro M, Invernici G, Cenci T, Maira G, Parati EA, Stassi G, Larocca LM and De Maria R: Tumour vascularization via endothelial differentiation of glioblastoma stem-like cells. Nature 468: 824-828, 2010.

8. Wang R, Chadalavada K, Wilshire J, Kowalik U, Hovinga KE, Geber A, Fligelman B, Leversha M, Brennan C and Tabar V: Glioblastoma stem-like cells give rise to tumour endothelium. Nature 468: 829-833, 2010

9. White E: The role for autophagy in cancer. J Clin Invest 125 : 42-46, 2015.

10. Mizushima N and Levine B: Autophagy in mammalian development and differentiation. Nat Cell Biol 12: 823-830, 2010.

11. Bronietzki AW, Schuster M and Schmitz I: Autophagy in T-cell development, activation and differentiation. Immunol Cell Biol 93: 25-34, 2015.

12. Zhang Y, Morgan MJ, Chen K, Choksi S and Liu ZG: Induction of autophagy is essential for monocyte-macrophage differentiation. Blood 119: 2895-2905, 2012.

13. Nuschke A, Rodrigues M, Stolz DB, Chu CT, Griffith L and Wells A: Human mesenchymal stem cells/multipotent stromal cells consume accumulated autophagosomes early in differentiation. Stem Cell Res Ther 5: 140, 2014.

14. Sugiyama M, Yoshizumi T, Yoshida Y, Bekki Y, Matsumoto Y, Yoshiya S, Toshima T, Ikegami T, Itoh S, Harimoto N, et al: p62 Promotes amino acid sensitivity of mTOR pathway and hepatic differentiation in adult liver stem/progenitor cells. J Cell Physiol 232: 2112-2124, 2017.

15. Shi X, Li W, Liu H, Yin D and Zhao J: $\beta$-Cyclodextrin induces the differentiation of resident cardiac stem cells to cardiomyocytes through autophagy. Biochim Biophys Acta Mol Cell Res 1864: 1425-1434, 2017.

16. Vazquez P, Arroba AI, Cecconi F, de la Rosa EJ, Boya P and de Pablo F: Atg5 and Ambra1 differentially modulate neurogenesis in neural stem cells. Autophagy 8: 187-199, 2012.

17. Bussolati B, Grange C, Sapino A and Camussi G: Endothelial cell differentiation of human breast tumour stem/progenitor cells. J Cell Mol Med 13: 309-319, 2009. 
18. Ghasemi A and Zahediasl S: Preanalytical and analytical considerations for meastutriing nitric oxide metabolites in serum or plasma using the griess method. Clin Lab 58: 615-624, 2012.

19. Livak KJ and Schmittgen TD: Analysis of relative gene expression data using real-time quantitative PCR and the 2(-Delta Delta $\mathrm{C}(\mathrm{T}))$ method. Methods 25: 402-408, 2001.

20. Ciurea ME, Georgescu AM, Purcaru SO, Artene SA, Emami GH, Boldeanu MV, Tache DE and Dricu A: Cancer stem cells: Biological functions and therapeutically targeting. Int J Mol Sci 15: 8169-8185, 2014

21. Kandel J, Bossy-Wetzel E, Radvanyi F, Klagsbrun M, Folkman J and Hanahan D: Neovascularization is associated with a switch to the export of bFGF in the multistep development of fibrosarcoma. Cell 66: 1095-1104, 1991.

22. Sturtzel C: Endothelial cells. Adv Exp Med Biol 1003: 71-91, 2017.

23. Parinandi NL, Sharma A, Eubank TD, Kaufman BF, Kutala VK, Marsh CB, Ignarro LJ and Kuppusamy P: Nitroaspirin (NCX-4016), an NO donor, is antiangiogenic through induction of loss of redox-dependent viability and cytoskeletal reorganization in endothelial cells. Antioxid Redox Signal 9: 1837-1849, 2007.

24. Deliu IC, Neagoe CD, Bezna M, Genunche-Dumitrescu AV, Toma SC, Ungureanu BS, Uscatu CD, Beznă MC, Lungulescu CV, Pădureanu V, et al: Correlations between endothelial cell markers CD31, CD34 and CD105 in colorectal carcinoma. Rom J Morphol Embryol 57: 1025-1030, 2016.

25. Klionsky DJ, Abdelmohsen K, Abe A, Abedin MJ, Abeliovich $\mathrm{H}$, Acevedo Arozena A, Adachi H, Adams CM, Adams PD, Adeli K, Adhihetty PJ, et al: Guidelines for the use and interpretation of assays for monitoring autophagy (3rd edition). Autophagy 12: $1-222,2016$.

26. Maycotte P, Aryal S, Cummings CT, Thorburn J, Morgan MJ and Thorburn A: Chloroquine sensitizes breast cancer cells to chemotherapy independent of autophagy. Autophagy 8: 200-212, 2012.

27. Wirth M, Joachim $\mathbf{J}$ and Tooze SA: Autophagosome formationthe role of ULK1 and Beclin1-PI3KC3 complexes in setting the stage. Semin Cancer Biol 23: 301-309, 2013.

28. Tanida I, Ueno $T$ and Uchiyama Y: Use of pHlurorinmKate2-human LC3 to monitor autophagic responses. Methods Enzymol 587: 87-96, 2017.

29. Ao LY, Li WT, Zhou L, Yan YY, Ye AQ, Liang BW, Shen WY, Zhu X and Li YM: Therapeutic effects of JLX-001 on ischemic stroke by inducing autophagy via AMPK-ULK1 signaling pathway in rats. Brain Res Bull 153: 162-170, 2019.

30. Al-Hajj M, Wicha MS, Benito-Hernandez A, Morrison SJ and Clarke MF: Prospective identification of tumorigenic breast cancer cells. Proc Natl Acad Sci USA 100: 3983-3988, 2003.

31. Kaur S and Bajwa P: A 'tete-a tete' between cancer stem cells and endothelial progenitor cells in tumor angiogenesis. Clin Transl Oncol 16: 115-121, 2014.
32. Fazioli F, Colella G, Miceli R, Di Salvatore MG, Gallo M, Boccella S, De Chiara A, Ruosi C and de Nigris F: Post-surgery fluids promote transition of cancer stem cell-to-endothelial and AKT/mTOR activity, contributing to relapse of giant cell tumors of bone. Oncotarget 8: 85040-85053, 2017.

33. Chroscinski D, Sampey D and Maherali N; Reproducibility Project: Cancer Biology; Reproducibility Project Cancer Biology: Registered report: Tumour vascularization via endothelial differentiation of glioblastoma stem-like cells. Elife 4, 2015.

34. El Hallani S, Boisselier B, Peglion F, Rousseau A, Colin C, Idbaih A, Marie Y, Mokhtari K, Thomas JL, Eichmann A, et al: A new alternative mechanism in glioblastoma vascularization: Tubular vasculogenic mimicry. Brain 133: 973-982, 2010.

35. Pattabiraman DR and Weinberg RA: Tackling the cancer stem cells-what challenges do they pose? Nat Rev Drug Discov 13: 497-512, 2014

36. Yang F, Xu J, Tang L and Guan X: Breast cancer stem cell: The roles and therapeutic implications. Cell Mol Life Sci 74: 951-966, 2017.

37. Liu G, Fan X, Tang M, Chen R, Wang H, Jia R, Zhou X, Jing W, Wang $\mathrm{H}$, Yang Y, et al: Osteopontin induces autophagy to promote chemo-resistance in human hepatocellular carcinoma cells. Cancer Lett 383: 171-182, 2016.

38. Hou J, Han ZP, Jing YY, Yang X, Zhang SS, Sun K, Hao C, Meng Y, Yu FH, Liu XQ, et al: Autophagy prevents irradiation injury and maintains stemness through decreasing ROS generation in mesenchymal stem cells. Cell Death Dis 4: e844, 2013.

39. Zhang J, Liu J, Huang Y, Chang JY, Liu L, McKeehan WL, Martin JF and Wang F: FRS2 $\alpha$-mediated FGF signals suppress premature differentiation of cardiac stem cells through regulating autophagy activity. Circ Res 110: E29-E39, 2012.

40. Takao S, Ding Q and Matsubara S: Pancreatic cancer stem cells: Regulatory networks in the tumor microenvironment and targeted therapy. J Hepatobiliary Pancreat Sci 19: 614-620, 2012.

41. Chun Y and Kim J: Autophagy: An essential degradation program for cellular homeostasis and life. Cells 7: E278, 2018.

42. Jiang S, Li T, Ji T, Yi W, Yang Z, Wang S, Yang Y and Gu C: AMPK: Potential therapeutic target for ischemic stroke. Theranostics 8: 4535-4551, 2018.

43. Wang Q, Bu S, Xin D, Li B, Wang L and Lai D: Autophagy is indispensable for the self-renewal and quiescence of ovarian cancer spheroid cells with stem cell-like properties. Oxid Med Cell Longev 2018: 7010472, 2018.

44. Shroff A and Reddy KVR: Autophagy gene ATG5 knockdown upregulates apoptotic cell death during Candida albicans infection in human vaginal epithelial cells. Am J Reprod Immunol 80: e13056, 2018.

45. Qian G, Liu D, Hou L, Hamid M, Chen X, Gan F, Song S and Huang K: Ochratoxin A induces cytoprotective autophagy via blocking AKT/mTOR signaling pathway in PK-15 cells. Food Chem Toxicol 122: 120-131, 2018.

46. Lee SG and Joe YA: Autophagy mediates enhancement of proangiogenic activity by hypoxia in mesenchymal stromal/stem cells. Biochem Biophys Res Commun 501: 941-947, 2018. 